\title{
Preclinical Evaluation of 3-18 F-Fluoro-2,2-Dimethylpropionic Acid as an Imaging Agent for Tumor Detection
}

\author{
Timothy H. Witney ${ }^{1}$, Federica Pisaneschi ${ }^{1}$, Israt S. Alam ${ }^{1}$, Sebastian Trousil ${ }^{1}$, Maciej Kaliszczak ${ }^{1}$, Frazer Twyman ${ }^{1}$, \\ Diana Brickute ${ }^{1}$, Quang-Dé Nguyen ${ }^{1}$, Zachary Schug ${ }^{2}$, Eyal Gottlieb ${ }^{2}$, and Eric O. Aboagye ${ }^{1}$ \\ ${ }^{I}$ Comprehensive Cancer Imaging Centre, Division of Cancer, Department of Surgery and Cancer, Imperial College London Faculty of \\ Medicine, London, United Kingdom; and ${ }^{2}$ Beatson Institute for Cancer Research, Glasgow, United Kingdom
}

\begin{abstract}
Deregulated cellular metabolism is a hallmark of many cancers. In addition to increased glycolytic flux, exploited for cancer imaging with ${ }^{18} \mathrm{~F}-\mathrm{FDG}$, tumor cells display aberrant lipid metabolism. Pivalic acid is a short-chain, branched carboxylic acid used to increase oral bioavailability of prodrugs. After prodrug hydrolysis, pivalic acid undergoes intracellular metabolism via the fatty acid oxidation pathway. We have designed a new probe, 3-18 F-fluoro-2,2-dimethylpropionic acid, also called ${ }^{18} \mathrm{~F}$-fluoro-pivalic acid $\left({ }^{18} \mathrm{~F}-\mathrm{FPIA}\right)$, for the imaging of aberrant lipid metabolism and cancer detection. Methods: Cell intrinsic uptake of ${ }^{18} \mathrm{~F}$-FPIA was measured in murine EMT6 breast adenocarcinoma cells. In vivo dynamic imaging, time course biodistribution, and radiotracer stability testing were performed. ${ }^{18} \mathrm{~F}-\mathrm{FPIA}$ tumor retention was further compared in vivo to ${ }^{18} \mathrm{~F}-\mathrm{FDG}$ uptake in several xenograft models and inflammatory tissue. Results: ${ }^{18} \mathrm{~F}-\mathrm{FPIA}$ rapidly accumulated in EMT6 breast cancer cells, with retention of intracellular radioactivity predicted to occur via a putative ${ }^{18} \mathrm{~F}-\mathrm{FPIA}$ carnitine-ester. The radiotracer was metabolically stable to degradation in mice. In vivo imaging of implanted EMT6 murine and BT474 human breast adenocarcinoma cells by ${ }^{18} \mathrm{~F}$-FPIA PET showed rapid and extensive tumor localization, reaching $9.1 \% \pm 0.5 \%$ and $7.6 \% \pm 1.2 \%$ injected dose/g, respectively, at 60 min after injection. Substantial uptake in the cortex of the kidney was seen, with clearance primarily via urinary excretion. Regarding diagnostic utility, uptake of ${ }^{18} \mathrm{~F}-\mathrm{FPIA}$ was comparable to that of ${ }^{18} \mathrm{~F}-\mathrm{FDG}$ in EMT6 tumors but superior in the DU145 human prostate cancer model (54\% higher uptake; $P=0.002)$. Furthermore, compared with ${ }^{18} \mathrm{~F}-\mathrm{FDG},{ }^{18} \mathrm{~F}-\mathrm{FPIA}$ had lower normal-brain uptake resulting in a superior tumor-to-brain ratio (2.5 vs. 1.3 in subcutaneously implanted U87 human glioma tumors; $P=0.001$ ), predicting higher contrast for brain cancer imaging. Both radiotracers showed increased localization in inflammatory tissue. Conclusion: ${ }^{18} \mathrm{~F}$-FPIA shows promise as an imaging agent for cancer detection and warrants further investigation.
\end{abstract}

Key Words: tumor detection; PET; FPIA; FDG; lipid metabolism

J Nucl Med 2014; 55:1506-1512

DOI: 10.2967/jnumed.114.140343

$\mathbf{T}$

umors reprogram their energy metabolism to support synthesis of proteins, lipids, and nucleotides required for growth, invasion,

Received Mar. 17, 2014; revision accepted May 19, 2014.

For correspondence or reprints contact: Eric Aboagye, Imperial College London, Room 242, MRC Cyclotron Building, Hammersmith Hospital, DuCane Rd., LondonW12 ONN, U.K.

E-mail: eric.aboagye@imperial.ac.uk

Published online Jul. 10, 2014.

COPYRIGHT (C 2014 by the Society of Nuclear Medicine and Molecular Imaging, Inc. and metastasis (1). The significantly increased glucose utilization of tumors in comparison to normal tissue (the Warburg effect), for instance, has been exploited clinically to detect tumors and their response to treatment by ${ }^{18} \mathrm{~F}-\mathrm{FDG}$ PET.

In addition to increased glycolytic flux, elevated de novo fatty acid synthesis and upregulated plasma membrane turnover in tumors, required for membrane synthesis during proliferation (2), have been traced with ${ }^{11} \mathrm{C}$ - and ${ }^{18} \mathrm{~F}$-labeled acetate and choline analogs (3-5). Although rates of glycogenesis, glutaminolysis, and fatty acid synthesis during the metabolic reprogramming of cancer cells have been comprehensively described, only recently has the role of fatty acid oxidation for cancer cell function been examined in detail (reviewed by Carracedo et al. (6)). Fatty acids provide an abundant energy source resulting from a cyclical series of reactions in the mitochondria that act to shorten the fatty acid and generate reduced nicotinamide adenine dinucleotide, reduced flavin adenine dinucleotide, and acetyl coenzyme A (CoA). The imaging of aberrant fatty acid oxidation by PET after malignant transformation has yet to be explored.

Short-chain carboxylates including acetate, propionate, butyrate, and the nonnatural substrate pivalate (trimethylacetate) use the early steps of the fatty acid oxidation pathway involving acyl-CoA and acyl-carnitine synthesis (7-9). Unlike acetate, however, pivalate cannot be oxidized to carbon dioxide in mammalian cells (10). We have designed a new probe, $3{ }^{18} \mathrm{~F}$-fluoro-2,2-dimethylpropionic acid, also called ${ }^{18} \mathrm{~F}$-fluoro-pivalic acid ( ${ }^{18} \mathrm{~F}$-FPIA; Fig. 1A), to image the early steps of the fatty acid oxidation pathway in cancer. We have recently reported the radiosynthesis of ${ }^{18}$ F-FPIA (11). In the present study, we evaluated the in vitro tumor cell uptake and metabolic stability of ${ }^{18}$ F-FPIA in tissues. We further investigated the potential utility of ${ }^{18}$ F-FPIA PET for tumor imaging in several xenograft models, in inflammatory tissue, and by comparison with ${ }^{18} \mathrm{~F}$-FDG PET.

\section{MATERIALS AND METHODS}

\section{Cell Culture}

EMT6 murine breast cancer cells (donation from Dr. Sofia Pascu, University of Bath, U.K.) were grown in Waymouth medium (Life Technologies), with U87 human glioma cells (LGC Standards) grown in Dulbecco modified Eagle medium (Life Technologies). DU145 (donation from Dr. Almut Schulze, CRUK London Research Institute, U.K.) and BT474 (LGC Standards) were grown in RPMI (Life Technologies). All media were supplemented with $2.5 \mathrm{~mL}$ of penicillin/ streptomycin $\left(100 \mathrm{IU} \cdot \mathrm{mL}^{-1} / 100 \mu \mathrm{g} \cdot \mathrm{mL}^{-1}\right)$ and $2 \mathrm{mM}$ L-glutamine (Life Technologies). Waymouth medium contained $15 \%$ fetal calf serum, with $10 \%$ fetal calf serum added to Dulbecco modified Eagle medium and RPMI. All cells were propagated at $37^{\circ} \mathrm{C}$ in a humidified atmosphere containing $5 \% \mathrm{CO}_{2}$. 


\section{Radiosynthesis}

Automated radiosynthesis of ${ }^{18}$ F-FPIA was based on previously described methodology (11), as detailed in the supplemental materials (available at http://jnm.snmjournals.org). ${ }^{18}$ F-FPIA was obtained in an
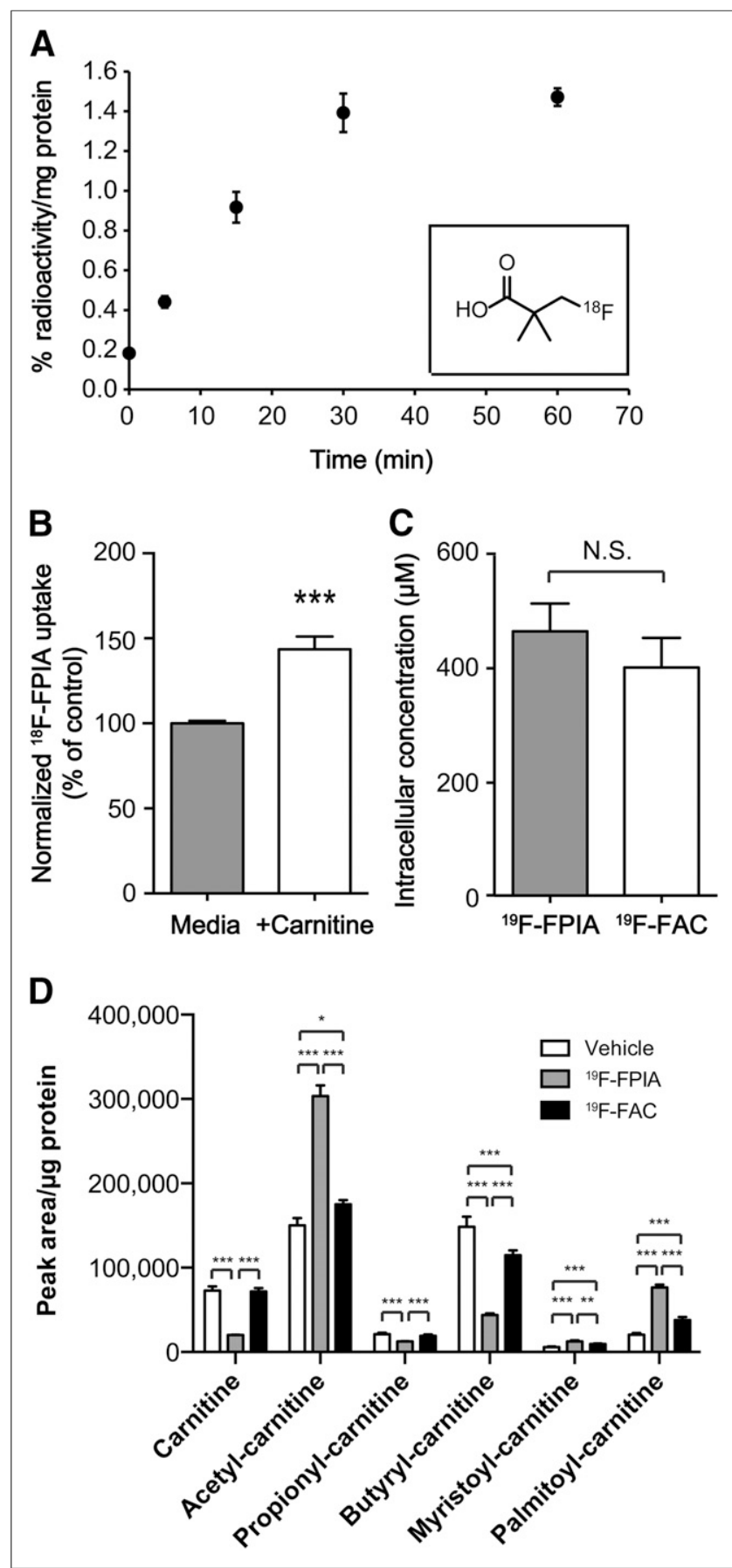

FIGURE 1. ${ }^{18} \mathrm{~F}-\mathrm{FPIA}$ uptake and metabolism in cancer cells. (A) Time course of ${ }^{18} \mathrm{~F}$-FPIA uptake in EMT6 cells $(n=3)$. Inset: chemical structure of ${ }^{18} \mathrm{~F}$-FPIA. (B) Effect of exogenous $10 \mu \mathrm{M}$ carnitine on ${ }^{18} \mathrm{~F}$-FPIA uptake, trapping, and retention in EMT6 cells $(n=3)$. (C) Intracellular accumulation of ${ }^{19} \mathrm{~F}$-FPIA and ${ }^{19} \mathrm{~F}-\mathrm{FAC}$ in human breast adenocarcinoma BT474 cells at $24 \mathrm{~h}$ as analyzed by liquid chromatography-mass spectrometry $(n=4)$. Effect of exogenous ${ }^{19} \mathrm{~F}-\mathrm{FPIA}$ and ${ }^{19} \mathrm{~F}-\mathrm{FAC}$ on intracellular metabolite concentrations of acyl-carnitine esters as analyzed by liquid chromatography-mass spectrometry $(n=4)$. Data are mean \pm SD. NS $=$ not statistically significant. end-of-synthesis yield of $7.7 \% \pm 5.0 \%(n=9)$ in approximately $90 \mathrm{~min}$ from aqueous fluoride to formulation with radiochemical purity of more than $99 \%$. Accurate and reliable measurement of specific activity was not possible by high-performance liquid chromatography because of the low ultraviolet absorbance of FPIA (11). ${ }^{18} \mathrm{~F}-\mathrm{FDG}$ was purchased from PETNET Solutions.

\section{Measurements in Cells}

EMT6 cells $\left(2 \times 10^{5}\right)$ were plated into 6-well plates overnight before ${ }^{18}$ F-FPIA uptake analysis. On the day of the experiment, fresh growth medium containing $0.74 \mathrm{MBq}$ of ${ }^{18} \mathrm{~F}$-FPIA was added to individual wells $(1 \mathrm{~mL} /$ well $)$. Cell uptake was measured over $60 \mathrm{~min}$ after radiotracer addition. Plates were placed on ice, washed 3 times with ice-cold phosphate-buffered saline (PBS), and lysed in radioimmunoprecipitation assay buffer (Thermo Fisher Scientific Inc.; $1 \mathrm{~mL}$, $10 \mathrm{~min}$ ). Cell lysates were transferred to counting tubes, and decaycorrected radioactivity was determined on a $\gamma$ counter (Cobra II AutoGamma counter; Packard Biosciences Co.). Aliquots were snap-frozen and used for protein determination after radioactive decay using a bicinchoninic acid 96-well plate assay (Thermo Fisher Scientific Inc.). In addition, $10 \mu \mathrm{L}$ standards from a $0.74 \mathrm{MBq} / \mathrm{mL}$ stock solution were counted to quantitate percentage radiotracer uptake. For carnitine treatment, cells were incubated with $10 \mu \mathrm{M}$ L-carnitine for the duration of the uptake time course.

For the assessment of cell uptake and resulting intracellular concentrations of ${ }^{19} \mathrm{~F}$-FPIA and ${ }^{19} \mathrm{~F}$-fluoroacetate $\left({ }^{19} \mathrm{~F}\right.$-FAC), BT474 cells were seeded in 12-well plates at $2 \times 10^{5}$ cells per well. A separate plate was seeded in parallel to determine the cell number and average intracellular volume per cell (Casy Cell Counter) and the amount of extraction solution to be added $\left(1 \mathrm{~mL} / 1 \times 10^{6}\right.$ cells $)$. The cells were treated with vehicle (dimethylsulfoxide), ${ }^{19} \mathrm{~F}$-FPIA, or ${ }^{19} \mathrm{~F}$-FAC (500 $\mu \mathrm{M})$ and incubated for $18 \mathrm{~h}$. The cells were washed once in $3 \mathrm{~mL}$ of ice-cold PBS, and intracellular metabolites were immediately extracted into a solution of methanol, acetonitrile, and water (5:3:2). Extracts were stirred in a vortex mixer for $10 \mathrm{~min}$ at $4{ }^{\circ} \mathrm{C}$ and centrifuged at $16,500 \mathrm{~g}$ at $4^{\circ} \mathrm{C}$ for $15 \mathrm{~min}$. Supernatants were removed and analyzed by liquid chromatography-mass spectrometry (ZIC-pHelic high-performance liquid chromatography columns and Exactive Plus Orbitrap MS; ThermoScientific). Data were analyzed using Xcalibur and LCQuan software (ThermoScientific). ${ }^{19} \mathrm{~F}$-FPIA and ${ }^{19} \mathrm{~F}$-FAC external standards were run in parallel and used to convert peak area to mass. In addition, after extraction, cells were dried to completion before protein measurement (12) to determine the relative changes in the abundance of intracellular metabolites.

\section{Measurements in Animal Models}

All animal experiments were performed by licensed investigators in accordance with the United Kingdom Home Office guidance on the operation of the Animals (Scientific Procedures) Act 1986 and within the published guidelines for the welfare and use of animals in cancer research (13). EMT6 tumor cells $\left(2 \times 10^{6} ; 100 \mu \mathrm{L}\right.$ of PBS $)$ were injected subcutaneously on the back of female BALB/c mice (aged 6-8 wk; Charles River) and grown to approximately $150 \mathrm{~mm}^{3}$. U87 tumors were grown after subcutaneous injection of $5 \times 10^{6}$ cells ( $100 \mu \mathrm{L}$ of PBS) on the back of female BALB/c nude mice (aged 6-8 wk; Charles River), with BT474 tumors induced after injection of $5 \times 10^{6}$ cells in Matrigel (BD Biosciences; 1:1 ratio of PBS to Matrigel; $100 \mu \mathrm{L}$ total). DU145 prostate adenocarcinoma xenografts were grown in male BALB/c nude mice (aged 6-8 wk; Charles River) after injection of $1 \times 10^{6}$ cells (1:1 PBS to Matrigel; $100 \mu \mathrm{L}$ ). U87, BT474, and DU145 tumors were grown to approximately $100 \mathrm{~mm}^{3}$ before analysis. Tumor dimensions were measured periodically using a caliper (by the same experienced researcher), and tumor volumes were calculated by the following equation: volume $=(\pi / 6) \times a \times b \times c$, where $a, b$, and $c$ represent 3 orthogonal 


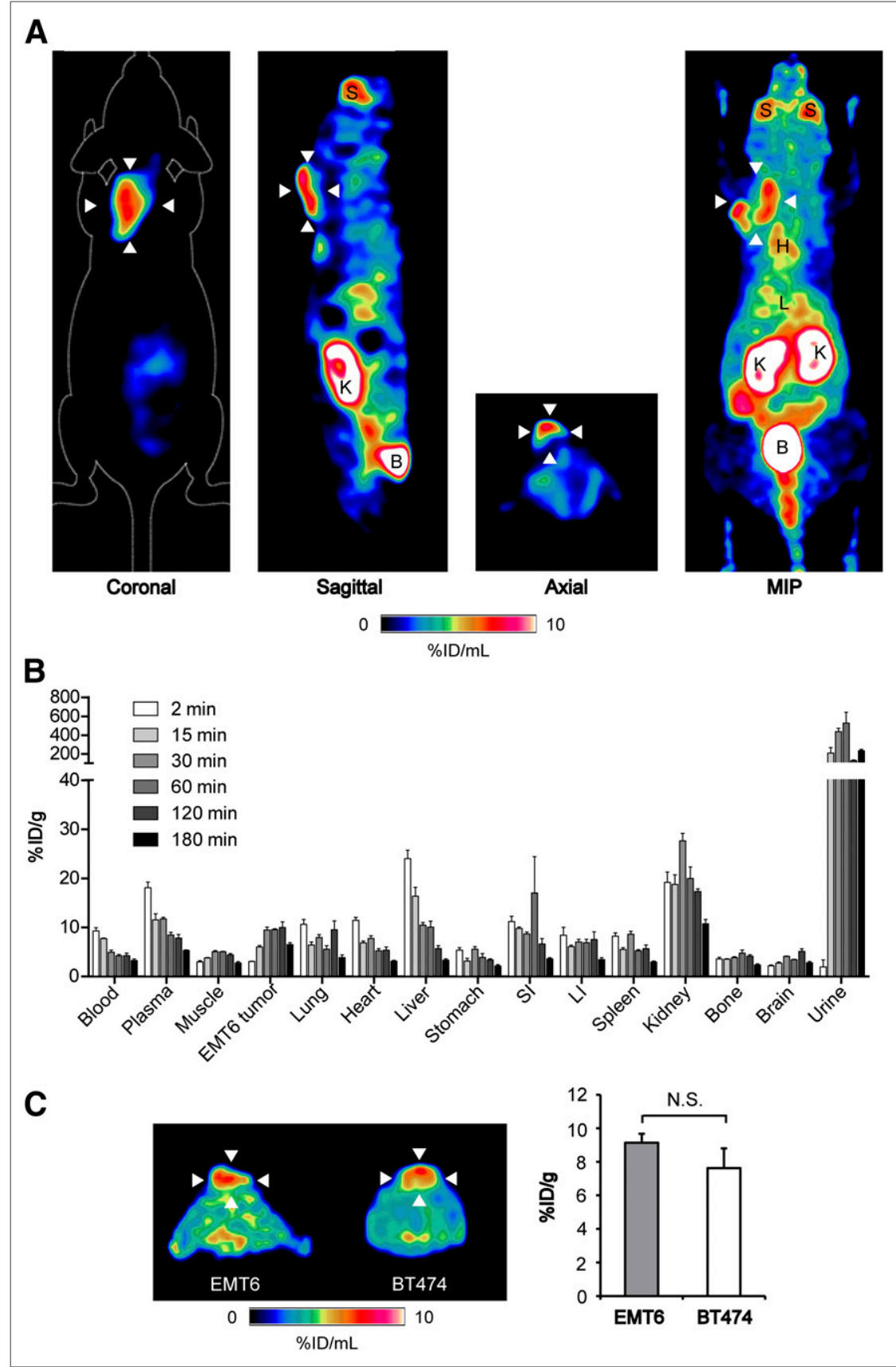

FIGURE 2. Time course ${ }^{18}$ F-FPIA pharmacokinetics and ${ }^{18}$ F-FPIA PET imaging of breast cancer xenografts. (A) Representative coronal, sagittal, axial, and maximum-intensity-projection PET images (10-min static scan) for ${ }^{18} \mathrm{~F}-\mathrm{FPIA}$ obtained $110-120 \mathrm{~min}$ after injection in EMT6 tumor-bearing mice. Arrowheads indicate tumor. (B) Time course ${ }^{18} \mathrm{~F}$-FPIA biodistribution performed at 2, 15, 30, 60, 120 , and $180 \mathrm{~min}$ after injection. Approximately $3.7 \mathrm{MBq}$ of ${ }^{18} \mathrm{~F}$-FPIA were injected into anesthetized EMT6 tumor-bearing BALB/c mice, which were sacrificed at the indicated time points. Data are mean $\pm \mathrm{SD}$ ( $n=3$ mice per group). (C) Comparison between ${ }^{18} \mathrm{~F}$-FPIA uptake in murine EMT6 and human BT474 breast tumors ( $n=4$ mice per group). $\mathrm{B}=$ bladder; $\mathrm{H}=$ heart; $\mathrm{K}=$ kidneys; $\mathrm{L}=$ liver; $\mathrm{LI}=$ large intestine; MIP = maximum intensity projection; $\mathrm{NS}=$ not statistically significant; $\mathrm{S}=$ salivary gland; $\mathrm{SI}=$ small intestine .

axes of the tumor (14). For these measurements, animals were kept in a warm environment to avoid skin folds that can confound measurements.

Inflammation was induced in an aseptic inflammation model, through intramuscular injection of turpentine oil $(30 \mu \mathrm{L})$ into the right posterior thigh of the mice, as previously described (15). For the analysis of radiotracer stability and metabolism, radiolabeled metabolites from

\section{RESULTS}

plasma and tissues were quantified using a semiautomated method adapted from Smith et al. (16), fully described in the supplemental materials.

For biodistribution studies, ${ }^{18}$ F-FPIA $(\sim 3.7$ $\mathrm{MBq})$ was injected via the tail vein of anesthetized EMT6 tumor-bearing BALB/c mice. The mice were maintained under anesthesia and sacrificed by exsanguination via cardiac puncture at $2,15,30,60,120$, or $180 \mathrm{~min}$ after radiotracer injection and tissues harvested $(n=3$ mice per group). Biodistribution studies were performed under anesthesia to permit comparison with dynamic imaging data. Biodistribution studies with BT474 and U87 tumor-bearing nude mice were performed $60 \mathrm{~min}$ after ${ }^{18} \mathrm{~F}$ FPIA injection. Tissue radioactivity was determined on a $\gamma$ counter (Cobra II Auto-Gamma counter) and decay-corrected. Ten-microliter standards from a 1:100 dilution of the stock activity were also counted for data normalization. Plasma was obtained from terminal blood samples after centrifugation $(6,000 \mathrm{~g}, 5 \mathrm{~min})$. Data were expressed as percentage injected dose per gram of tissue $(\% \mathrm{ID} / \mathrm{g})$.

${ }^{18}$ F-FPIA images were obtained on a GENISYS $^{4}$ small-animal PET scanner (Sofie Biosciences), after a bolus intravenous injection of approximately $1.85 \mathrm{MBq}$ of the radiotracer into tumor-bearing mice. Static scans were acquired for $10 \mathrm{~min}$ (at $50-60 \mathrm{~min}$ or 110-120 $\mathrm{min}$ ), and images were reconstructed using maximum-likelihood expectation maximization (matrix size, $208 \times 96 \times 96$ ). In a different cohort of mice, dynamic PET imaging (binned into the following frames: $15 \mathrm{~s} \times 4$, $60 \mathrm{~s} \times 4$, and $300 \mathrm{~s} \times 11$ ) was performed on an Inveon PET/SPECT/CT scanner (Siemens Medical Solutions; matrix size, $256 \times 256 \times$ $159)$ as described in detail in the supplemental materials. Dynamic PET imaging was performed for the ${ }^{18} \mathrm{~F}$-FPIA and ${ }^{18} \mathrm{~F}-\mathrm{FDG}$ comparison study in EMT6 tumor-bearing mice, with 50- to 60-min cumulative images of the dynamic data used for image reconstruction. Static scans on the GENISYS ${ }^{4}$ were used for all other experiments $(n=4$ mice per group). Comparable performance of the 2 scanners has previously been established (17). Inveon Research Workplace software was used to visualize radiotracer uptake in the tumor and to manually define the 3-dimensional volumes of interest. Tumor radioactivity was normalized to that of the whole body to obtain the normalized uptake value (NUV) to permit comparison of data obtained using the 2 scanners.

Tumor Cell Uptake and Metabolism

Uptake of ${ }^{18}$ F-FPIA into EMT6 tumor cells was linear over the initial $30 \mathrm{~min}$ of incubation, reaching $1.40 \% \pm 0.10 \%$ radioactivity per milligram of protein. By 60 min after radiotracer addition, cell 
TABLE 1

Time Course ${ }^{18}$ F-FPIA Tumor-to-Tissue Ratios in EMT6 Xenografts

\begin{tabular}{ccccccc}
\hline & \multicolumn{6}{c}{ Time after injection (min) } \\
\cline { 2 - 7 } Organ & 2 & 15 & 30 & 60 & 120 & 180 \\
\hline Tumor-to-muscle & 1.03 & 1.62 & 1.91 & 1.93 & 2.32 & 2.06 \\
\hline Tumor-to-blood & 0.32 & 0.77 & 1.96 & 2.32 & 2.41 & 2.45
\end{tabular}

Data are mean $(n=3)$.

uptake had plateaued at $1.47 \% \pm 0.04 \%$ radioactivity per milligram of protein (Fig. 1A). ${ }^{18}$ F-FPIA uptake increased by $44 \%$ compared with control cells after incubation with $10 \mu \mathrm{M} \mathrm{L}$-carnitine (Fig. 1B), suggesting possible use of carnitine for esterification of ${ }^{18}$ F-FPIA, although other mechanisms, for example, inhibition of acyl-carnitine efflux transporters, could be at play.

Unlabeled ${ }^{19}$ F-FPIA was also avidly taken up by BT474 cells, detected by mass spectrometry (Fig. 1C). At the higher probe concentrations used (500 $\mu \mathrm{M}$ in medium), we confirmed the presence of intracellular ${ }^{19} \mathrm{~F}$-FPIA; however, no other metabolites were observed. The presence of a CoA derivative of ${ }^{19} \mathrm{~F}$-FPIA could not be ruled out, since in control experiments with ${ }^{19} \mathrm{~F}-\mathrm{FAC}$, only the parent

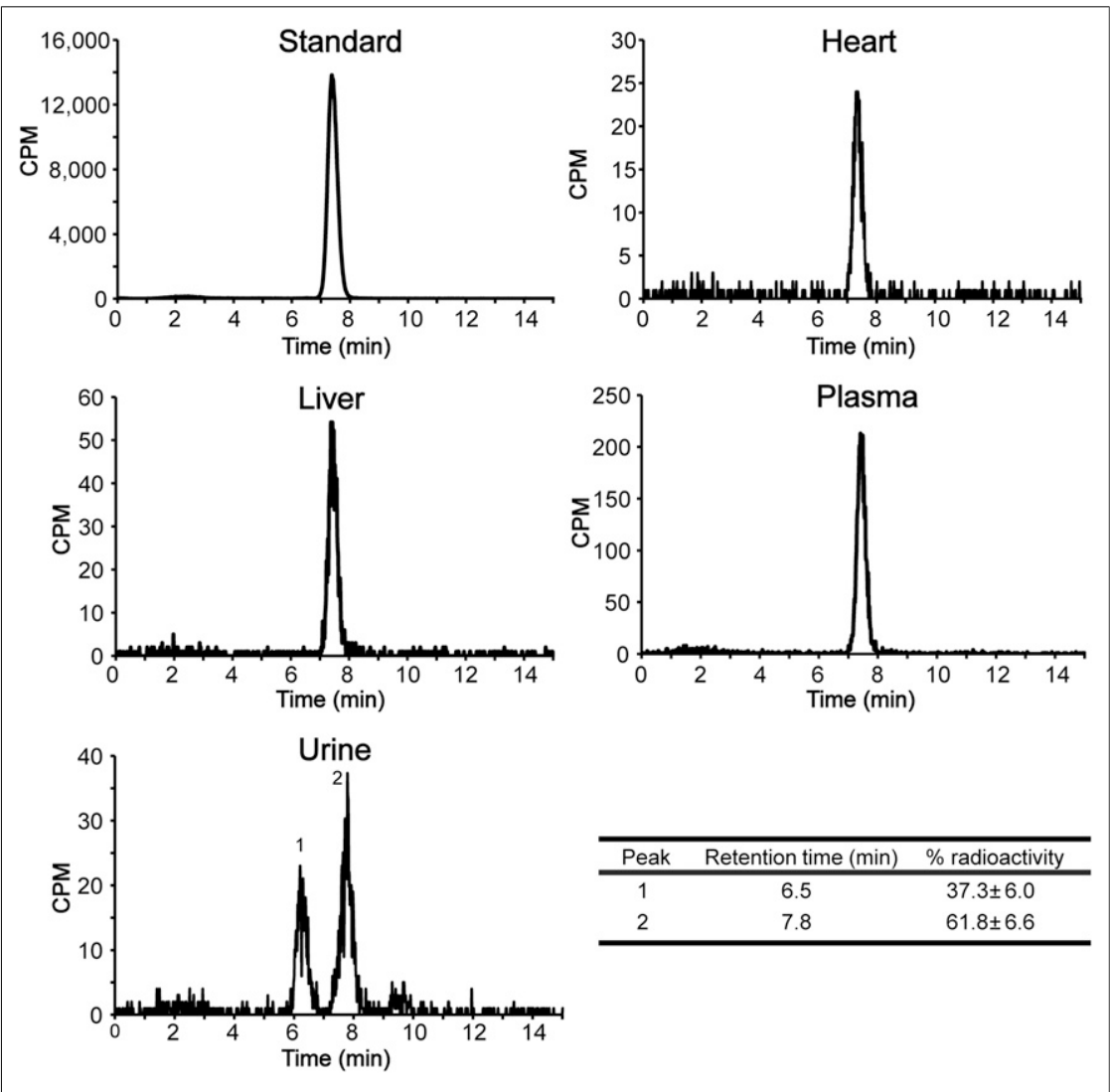

FIGURE 3. Representative radio-high-performance liquid chromatography analysis of mouse tissue extracts. Tissues were obtained $30 \mathrm{~min}$ after intravenous injection of ${ }^{18} \mathrm{~F}-\mathrm{FPIA}$ into nontumor-bearing BALB/c mice and compared with injection-ready ${ }^{18} \mathrm{~F}-\mathrm{FPIA}$ as standard. Percentage urine radioactivity of ${ }^{18} \mathrm{~F}$-FPIA (peak 2; retention time, $7.8 \mathrm{~min}$ ) and unknown metabolite (peak 1; retention time, $6.5 \mathrm{~min})$ are shown. Data are mean $\pm \mathrm{SD}(n=3$ mice). Table shows urine metabolite analysis. compound and ${ }^{19} \mathrm{~F}$-fluorocitrate were observed (Fig. 1C and Supplemental Fig. 1A, respectively), which implies conversion via fluoroacetyl-CoA. Intracellular levels of ${ }^{19}$ F-FPIA were similar to those of ${ }^{19} \mathrm{~F}-\mathrm{FAC}$ and its derived compounds (Fig. 1C). In addition, ${ }^{19} \mathrm{~F}$-FAC but not ${ }^{19}$ F-FPIA caused negative feedback inhibition in cells (a significant increase in citrate and cis-aconitate, together with a significant decrease in $\alpha$-ketoglutarate due to inhibition of aconitase; Supplemental Figs. 1B-1D). In contrast, ${ }^{19}$ F-FPIA produced a strong and specific reduction of intracellular nonesterified carnitine (Fig. 1D). ${ }^{19} \mathrm{~F}$-FAC did not decrease intracellular carnitine, demonstrating differences between these 2 compounds. Furthermore, ${ }^{19}$ F-FPIA showed specific induction of acetylcarnitine, myristoylcarnitine, and palmitoylcarnitine but a decrease in medium-chain acylcarnitines (Fig. 1D and Supplemental Fig. 2). Conversely, medium-chain acylcarnitines were increased after ${ }^{19} \mathrm{~F}$-FAC treatment (Supplemental Fig. 2).

\section{Measurements In Vivo}

We next evaluated ${ }^{18}$ F-FPIA for in vivo tumor imaging. Substantial ${ }^{18}$ F-FPIA tumor localization was measured by PET in EMT6 murine breast adenocarcinoma xenografts, clearly visible above background normal tissue. High ${ }^{18}$ F-FPIA uptake was also observed in the bladder, kidney, and salivary glands. Representative static ${ }^{18} \mathrm{~F}$ FPIA scans showing the 3 orthogonal axes of EMT6 tumor-bearing mice and an associated maximum-intensity projection 110$120 \mathrm{~min}$ after injection are shown in Figure 2A, with the time course biodistribution of ${ }^{18}$ F-FPIA over 180 min illustrated in Figure 2B. Similar to in vitro cell uptake, tumorassociated radioactivity linearly increased over the initial $30 \mathrm{~min}$ after injection to $9.3 \pm 1.0 \% \mathrm{ID} / \mathrm{g}$, followed by stable retention in the tumor up to $120 \mathrm{~min}$ after radiotracer injection $(9.8 \pm 2.0 \% \mathrm{ID} / \mathrm{g})$. By $180 \mathrm{~min}$, tumor-associated ${ }^{18} \mathrm{~F}$-FPIA reduced $36 \%$ to $6.3 \pm 0.7 \% \mathrm{ID} / \mathrm{g} .{ }^{18} \mathrm{~F}$-FPIA pharmacokinetics were characterized by initial liver uptake, followed by rapid clearance, with the urinary tract being the primary route of excretion, determined both by biodistribution (Fig. 2B) and from tissue time-activity curves derived from dynamic imaging studies (Supplemental Fig. 3). Optimum tumor-toblood and tumor-to-muscle ratios were achieved at $120 \mathrm{~min}$ after injection, at 2.4 and 2.3 , respectively (Table 1 ), although these were not substantially different from those at $60 \mathrm{~min}$ (1.9 and 2.3, respectively). For practical reasons (including the comfort of subjects), $60 \mathrm{~min}$ was used for further dynamic and static imaging studies. Furthermore, tumor radioactivity was comparable for both the murine and the human breast adenocarcinoma tumor models, reaching $9.1 \pm 0.5 \% \mathrm{ID} / \mathrm{g}$ and $7.6 \pm 1.2 \% \mathrm{ID} / \mathrm{g}$ at 60 min after injection in EMT6 and BT474 tumors, respectively (Fig. 2C).

\section{Radiotracer Stability In Vivo}

To confirm that tissue-associated radioactivity corresponded with parent ${ }^{18}$ F-FPIA, 


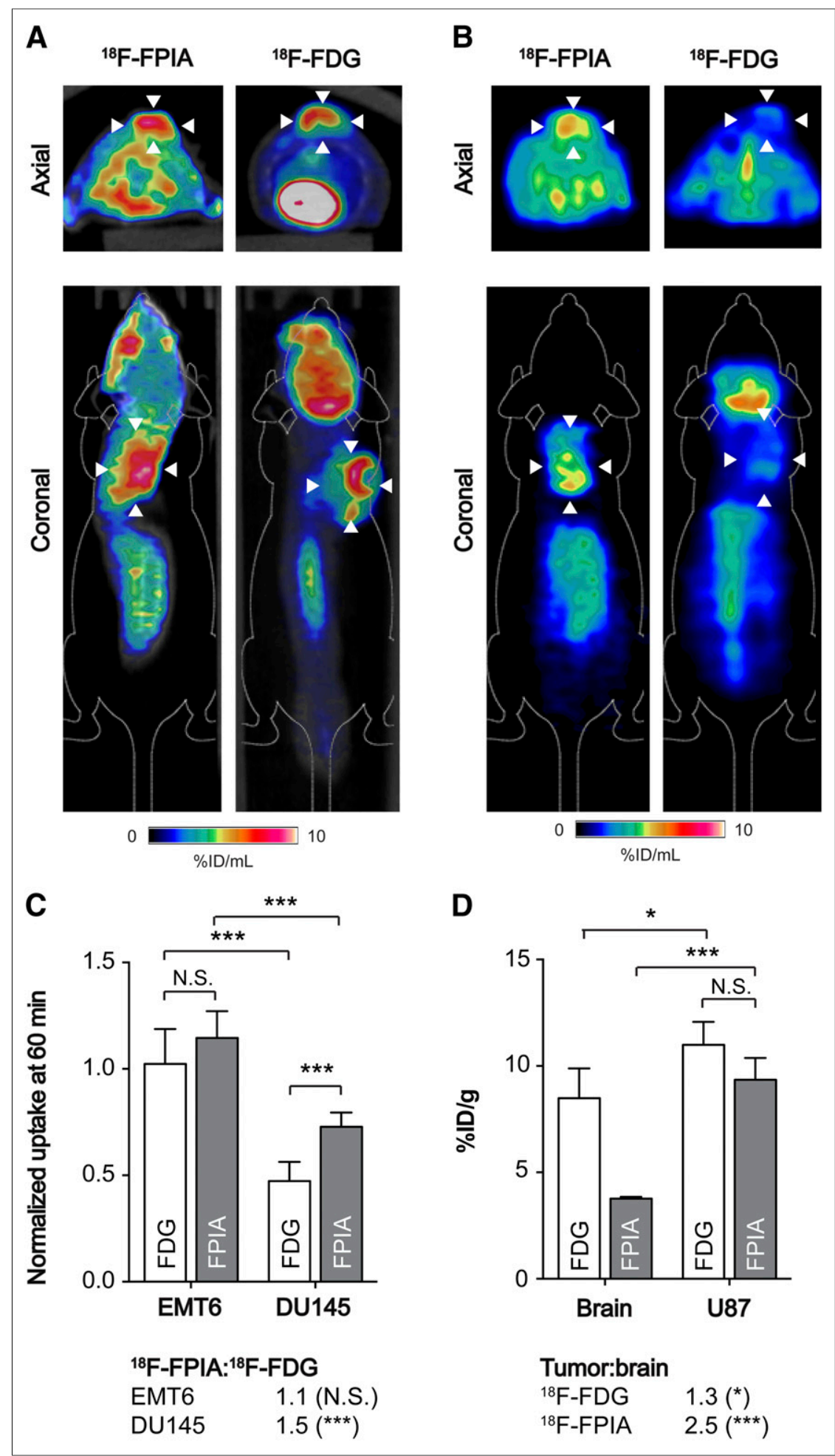

FIGURE 4. Tumor imaging with ${ }^{18} \mathrm{~F}$-FPIA and ${ }^{18} \mathrm{~F}$-FDG PET. (A) Representative axial and coronal PET/CT images (50-60 min of summed activity) for ${ }^{18} \mathrm{~F}$-FPIA and ${ }^{18} \mathrm{~F}-\mathrm{FDG}$ in EMT6 tumorbearing mice. Arrowheads indicate tumor, identified from CT image. (B) Representative axial and coronal PET images of DU145 tumor-bearing mice (50-60 min of summed activity) for ${ }^{18}$ F-FPIA and ${ }^{18}$ F-FDG. (C) Semiquantitative tumor uptake values for DU145 and EMT6, extracted from PET images and normalized to whole-body radioactivity. Data are mean \pm SD $(n=4-6$ mice per group). (D) ${ }^{18} \mathrm{~F}$-FPIA and ${ }^{18} \mathrm{~F}-\mathrm{FDG}$ biodistribution in U87 tumor-bearing mice. Approximately 3.7 MBq of ${ }^{18} \mathrm{~F}$-FPIA were injected into anesthetized BALB/c mice, which were sacrificed 60 min after injection. Data are mean \pm SD $\left(n=4\right.$ mice per group). ${ }^{\star} P<0.05{ }^{* \star \star} P<0.001$. NS $=$ not statistically significant. rather than undesired degradation products, ${ }^{18}$ F-FPIA stability was tested in tissues. ${ }^{18} \mathrm{~F}$ FPIA showed good stability in plasma, liver, and heart at $30 \mathrm{~min}$ after injection, with only the peaks of the parent compound detectable by radio-high-performance liquid chromatography (Fig. 3). In the urine, the parent peak was the dominant fraction at 30 min (61.8\% $\pm 6.6 \%$ radioactivity), with an additional unidentified metabolite peak observed at $6.5 \mathrm{~min}(37.3 \% \pm 6.0 \%$ radioactivity).

\section{Comparison of ${ }^{18}$ F-FPIA to ${ }^{18}$ F-FDG} for Tumor Detection

Given the promising ${ }^{18}$ F-FPIA uptake in EMT6 and BT474 tumors, dynamic ${ }^{18}$ F-FPIA PET imaging was performed over $60 \mathrm{~min}$ and compared with the gold-standard PET tracer for tumor diagnosis, ${ }^{18} \mathrm{~F}$-FDG; the same scanner was used for both radiotracers. High tumor uptake was detected by PET for both ${ }^{18} \mathrm{~F}$-FPIA and ${ }^{18} \mathrm{~F}$-FDG in EMT6 tumors, illustrated in 50- to 60-min images (Fig. $4 \mathrm{~A})$. In contrast, DU145 prostate adenocarcinoma tumors were clearly discernible by ${ }^{18}$ F-FPIA PET, whereas tumor-associated radioactivity with ${ }^{18} \mathrm{~F}-\mathrm{FDG}$ was not visible above background in 50- to 60-min static scans (Fig. 4B). For EMT6 tumors, tumorto-whole-body normalized uptake reached $1.02 \pm 0.16 \mathrm{NUV}$ at $60 \mathrm{~min}$ for ${ }^{18} \mathrm{~F}-\mathrm{FDG}$, compared with $1.15 \pm 0.13$ for ${ }^{18} \mathrm{~F}$-FPIA (Fig. 4C; $P=0.08 ; n=4-6$ ). Semiquantitative imaging parameters derived from the tumor time-activity curve also could not differentiate between these 2 tracers (Supplemental Fig. 4). Although lower than for EMT6 tumors, ${ }^{18}$ F-FPIA tumor uptake was $54 \%$ higher than ${ }^{18} \mathrm{~F}$-FDG in DU145 prostate tumors, at $0.73 \pm 0.07$ and $0.47 \pm 0.09$ NUV, respectively (Fig. 4C; $P=0.002$; $n=4-5)$.

Given that ${ }^{18}$ F-FPIA passes the blood-brain barrier, shown by $\gamma$ counting (Fig. 2B), we next tested the potential to use ${ }^{18}$ F-FPIA to detect tumors of the brain, comparing uptake values to ${ }^{18}$ F-FDG using the well-characterized U87 glioma model. In these humanderived xenografts, similarly high ${ }^{18}$ F-FPIA and ${ }^{18}$ F-FDG tumor uptake was measured $60 \mathrm{~min}$ after radiotracer injection, at $9.35 \pm$ $1.0 \% \mathrm{ID} / \mathrm{g}$ and $11.0 \pm 1.1 \% \mathrm{ID} / \mathrm{g}$, respectively (Fig. 4D; $P=0.07 ; n=4$ ). However, because of its low background retention, ${ }^{18} \mathrm{~F}$ FPIA provided a significantly higher tumorto-brain ratio of 2.5, compared with just 1.3 with ${ }^{18} \mathrm{~F}-\mathrm{FDG}(P=0.001 ; n=4)$.

Inflammatory cells are known to display both elevated glycolysis and fatty acid oxidation (18). Given this, we explored 


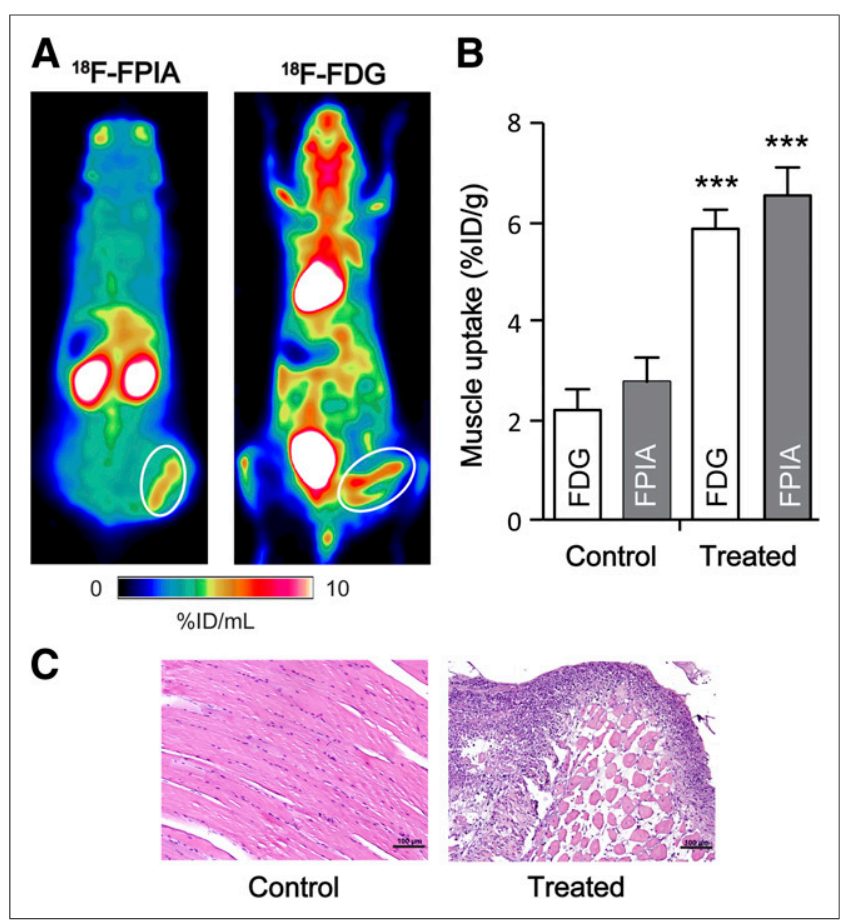

FIGURE 5. ${ }^{18} \mathrm{~F}-\mathrm{FPIA}$ and ${ }^{18} \mathrm{~F}-\mathrm{FDG}$ uptake in aseptic inflammation model. (A) Representative coronal PET images (30- to 60-min summed frames) for ${ }^{18} \mathrm{~F}$-FPIA and ${ }^{18} \mathrm{~F}$-FDG. Turpentine-induced inflammatory tissue is circled in white. (B) ${ }^{18} \mathrm{~F}-\mathrm{FPIA}$ and ${ }^{18} \mathrm{~F}-\mathrm{FDG}$ biodistribution of turpentinetreated and control, untreated posterior thigh muscle. Data are mean \pm SD ( $n=3-4)$. (C) Immunohistochemistry analysis by hematoxylin and eosin staining in control and turpentine-treated muscle. Representative photographs of stained sections were acquired at $\times 200$ magnification. Scale bar $=100 \mu \mathrm{m}$. ${ }^{\star \star \star} P<0.001$.

${ }^{18}$ F-FPIA and the known ability of ${ }^{18}$ F-FDG to image this condition using an aseptic inflammation model. Both ${ }^{18} \mathrm{~F}$-FPIA and ${ }^{18} \mathrm{~F}$ FDG accumulated in chemically induced inflammatory tissues, at $6.53 \pm 1.13 \% \mathrm{ID} / \mathrm{g}$ and $5.86 \pm 0.68 \% \mathrm{ID} / \mathrm{g}$, respectively (Fig. 5). There was no significant difference between inflammation-to-muscle ratios for ${ }^{18}$ F-FPIA (2.36) and ${ }^{18}$ F-FDG (2.66). Inflammation was confirmed by histologic analysis after imaging (Fig. 5C).

\section{DISCUSSION}

Pivalic acid is a short-chain carboxylic acid, containing a gemdimethyl group, and structural analog of propionate. Pivalic acid pharmacokinetics and intracellular metabolism at pharmacologic doses have been well described in the literature as the result of the widespread use of pivalic acid-containing prodrugs (reviewed by Brass (10)). Pivalate is intracellularly activated by acyl-CoA synthetases to pivaloyl-CoA, which, like other short-chain fatty acids including acetate and propionate, is transient in cells $(7,8,19)$. Oxidation to $\mathrm{CO}_{2}$ is not possible in mammalian cells. Instead, transesterification of pivaloyl-CoA to pivaloyl-carnitine occurs under the action of carnitine acyl transferases (20). Pivaloyl-carnitine is membrane-impermeable and is transported out of tissues via the human kidney carnitine transporter, OCTN2, whose transport is competitively inhibited by L-carnitine (21). In this study, we used trace quantities of pivalic acid labeled with ${ }^{18} \mathrm{~F}\left({ }^{18} \mathrm{~F}\right.$-FPIA) and investigated its behavior in vivo as a possible PET tracer for cancer imaging.
The increased uptake of ${ }^{18}$ F-FPIA after incubation with L-carnitine warranted further investigation of putative ${ }^{18} \mathrm{~F}$-FPIA CoA or carnitine ester. Extensive work to synthesize the ${ }^{19}$ F-FPIA CoA or carnitine ester reference material was unsuccessful. Using mass spectrometry, we confirmed intracellular levels of ${ }^{18}$ F-FPIA, but again we could not rule out the existence of metabolites by comparison to ${ }^{19} \mathrm{~F}-\mathrm{FAC}$. Supporting these data, carnitine esters have previously been shown to be major metabolic products of both ${ }^{13} \mathrm{C}$-acetate and ${ }^{13} \mathrm{C}$-propionate catabolism in the heart, measured over a far shorter experimental window of $70 \mathrm{~s}$ by hyperpolarized ${ }^{13} \mathrm{C}$ MR spectroscopy-with CoA esters a minor metabolic product (19). Here, ${ }^{19}$ F-FPIA treatment of cells resulted in a striking alteration in the levels of carnitine and associated acylcarnitine esters when compared with vehicle control (Fig. 1D). This observation is in keeping with the finding of profound carnitine deficiency as a result of pivalate toxicity in patients (22) and provides a direct link between FPIA and fatty acid oxidation. We speculate that decreases in mediumchain acylcarnitines could be due to inhibition of medium-chain acylcarnitine transferases by FPIA or FPIA esters (medium-chain species). The inhibitory effect on medium-chain acylcarnitines might have led to increased short-chain and long-chain acylcarnitines in order to maintain a normal fatty acid oxidation status. This hypothesis, however, requires further testing. Thus, whereas the mechanism of ${ }^{18}$ F-FPIA trapping is likely to occur via CoA and carnitine esters, we have not conclusively proven that in the work reported here.

Time course biodistribution studies revealed organ-specific variations in ${ }^{18}$ F-FPIA retention and pharmacokinetics, characterized by initial uptake in the liver and clearance primarily through the urinary tract and, to a lesser extent, hepatobiliary elimination; assessment of metabolism in intestinal contents will further clarify the nature of the radioactive species in this tissue. In agreement with previously published data on carnitine-acylcarnitine distribution (23), liver-associated ${ }^{18}$ F-FPIA rapidly equilibrated with the plasma compartment. The relatively high overall plasma concentration ( 5-18\% ID/g over 2-180 min) and plasma half-life (20.2 $\mathrm{min}$ ) of ${ }^{18}$ F-FPIA observed here is almost certainly accounted for by the high reabsorption rate of small-chain fatty acids by the proximal tubules (24), with FPIA and its metabolites excreted into the urine (Fig. 3). The distribution profile of ${ }^{18} \mathrm{~F}$-FPIA in other organs was also in keeping with the known tissue pharmacokinetics of pivalic acid in rodents (8). Of note, our study with ${ }^{18}$ F-FPIA (both biodistribution and imaging) was done under anesthesia, and such should be kept in mind during interpretation of the study results.

We further showed that ${ }^{18}$ F-FPIA preferentially accumulates in murine and human tumors of the breast, brain, and prostate used in this study. We speculate that upregulation of enzymatic activity (e.g., acetyl CoA synthetase and carnitine acetyltransferase), decreased OCTN2 expression, or elevated pools of metabolic intermediates may account for increased tumor-to-normal tissue retention. High serum carnitine ( $\sim 60 \mu \mathrm{M}$ in healthy adult men (25)) may further enhance ${ }^{18}$ F-FPIA tumor retention, analogous to the increased uptake measured in cell experiments after addition of exogenous carnitine. We compared tumoral ${ }^{18}$ F-FPIA uptake with ${ }^{18}$ F-FDG uptake and retention. Although it was impossible to differentiate between the 2 radiotracers by NUVs in the breast adenocarcinoma xenografts, ${ }^{18}$ F-FPIA radiotracer retention was $54 \%$ higher in the prostate xenografts than with ${ }^{18} \mathrm{~F}-\mathrm{FDG}$, indicating the potential utility for ${ }^{18}$ F-FPIA for prostate cancer detection. Voiding of bladder radioactivity should enable good prostate 
tumor delineation, given the urinary excretion profile of ${ }^{18} \mathrm{~F}$-FPIA. Further work is required to assess the effect of grade or androgen receptor status on ${ }^{18} \mathrm{~F}$-FPIA uptake. We also observed that ${ }^{18} \mathrm{~F}$-FPIA uptake was significantly lower than ${ }^{18} \mathrm{~F}$-FDG uptake in the normal brain. The improved human glioma tumor-to-brain ratio of 2.5 for ${ }^{18} \mathrm{~F}$-FPIA, versus 1.3 for ${ }^{18} \mathrm{~F}$-FDG, could be advantageous for ${ }^{18} \mathrm{~F}$ FPIA visualization of brain tumors when compared with ${ }^{18} \mathrm{~F}$-FDG. One limitation of ${ }^{18} \mathrm{~F}$-FPIA is that it does not appear to provide an advantage over ${ }^{18}$ F-FDG for the differential diagnosis of cancer versus inflammation (Fig. 5) (26). This warrants further studies of this radiotracer in nonmalignant inflammation-related pathologies, including Alzheimer's disease and multiple sclerosis.

\section{CONCLUSION}

${ }^{18}$ F-FPIA was designed for the imaging of aberrant lipid metabolism associated with malignant transformation. The radiotracer showed high accumulation in breast, prostate, and brain tumor models-accumulation comparable or superior to that of ${ }^{18} \mathrm{~F}$ FDG. The lower normal-brain uptake (and hence the inferred superior tumor-to-brain contrast) is a promising characteristic for brain tumor imaging. Future work will further investigate the underlying molecular mechanisms that govern ${ }^{18}$ F-FPIA uptake and evaluate its potential for orthotopic brain tumor imaging before clinical translation.

\section{DISCLOSURE}

The costs of publication of this article were defrayed in part by the payment of page charges. Therefore, and solely to indicate this fact, this article is hereby marked "advertisement" in accordance with 18 USC section 1734. This work was funded by a Cancer Research U.K. Discovery award (C11589/A13222). A patent on this invention (application number: PCT/GB2014/051405) has been filed. No other potential conflict of interest relevant to this article was reported.

\section{ACKNOWLEDGMENTS}

We thank Dr. Almut Schulze for donation of DU145 cells, Dr. Sofia Pascu for EMT6 cells, and Dr. Laurence Carroll for technical assistance.

\section{REFERENCES}

1. Cairns RA, Harris IS, Mak TW. Regulation of cancer cell metabolism. Nat Rev Cancer. 2011;11:85-95.

2. Baenke F, Peck B, Miess H, Schulze A. Hooked on fat: the role of lipid synthesis in cancer metabolism and tumour development. Dis Model Mech. 2013;6:1353-1363.
3. Oyama N, Akino H, Kanamaru $\mathrm{H}$, et al. ${ }^{11} \mathrm{C}$-acetate PET imaging of prostate cancer. J Nucl Med. 2002;43:181-186.

4. Ponde DE, Dence CS, Oyama N, et al. ${ }^{18} \mathrm{~F}$-fluoroacetate: a potential acetate analog for prostate tumor imaging-in vivo evaluation of ${ }^{18} \mathrm{~F}$-fluoroacetate versus ${ }^{11}$ C-acetate. J Nucl Med. 2007;48:420-428.

5. Witney TH, Alam IS, Turton DR, et al. Evaluation of deuterated F-18- and C-11labeled choline analogs for cancer detection by positron emission tomography. Clin Cancer Res. 2012;18:1063-1072.

6. Carracedo A, Cantley LC, Pandolfi PP. Cancer metabolism: fatty acid oxidation in the limelight. Nat Rev Cancer. 2013;13:227-232.

7. Bastiaansen JA, Cheng T, Mishkovsky M, Duarte JM, Comment A, Gruetter R. In vivo enzymatic activity of acetylCoA synthetase in skeletal muscle revealed by ${ }^{13} \mathrm{C}$ turnover from hyperpolarized $\left[1-{ }^{13} \mathrm{C}\right]$ acetate to $\left[1-{ }^{13} \mathrm{C}\right]$ acetylcarnitine. Biochim Biophys Acta. 2013;1830:4171-4178.

8. Diep QN, Brors O, Bohmer T. Formation of pivaloylcarnitine in isolated rat heart cells. Biochim Biophys Acta. 1995;1259:161-165.

9. Ruff LJ, Brass EP. Metabolic effects of pivalate in isolated rat hepatocytes. Toxicol Appl Pharmacol. 1991;110:295-302.

10. Brass EP. Pivalate-generating prodrugs and carnitine homeostasis in man. Pharmacol Rev. 2002;54:589-598.

11. Pisaneschi F, Witney TH, Iddon L, Aboagye EO. Synthesis of [F-18]fluoropivalic acid: an improved PET imaging probe for the fatty acid synthesis pathway in tumours. Medchemcomm. 2013;4:1350-1353.

12. Lowry OH, Rosebrough NJ, Farr AL, Randall RJ. Protein measurement with the Folin phenol reagent. J Biol Chem. 1951;193:265-275.

13. Workman P, Aboagye EO, Balkwill F, et al. Guidelines for the welfare and use of animals in cancer research. Br J Cancer. 2010;102:1555-1577.

14. Tomayko MM, Reynolds CP. Determination of subcutaneous tumor size in athymic (nude) mice. Cancer Chemother Pharmacol. 1989;24:148-154.

15. Witney TH, Carroll L, Alam IS, et al. A novel radiotracer to image glycogen metabolism in tumors by positron emission tomography. Cancer Res. 2014;74: 1319-1328.

16. Smith G, Zhao Y, Leyton J, et al. Radiosynthesis and pre-clinical evaluation of $\left[{ }^{18} \mathrm{~F}\right]$ fluoro-[1,2- $\left.{ }^{2} \mathrm{H}_{4}\right]$ choline. Nucl Med Biol. 2011;38:39-51.

17. Herrmann K, Dahlbom M, Nathanson D, et al. Evaluation of the Genisys4, a bench-top preclinical PET scanner. J Nucl Med. 2013;54:1162-1167.

18. O'Neill LA, Hardie DG. Metabolism of inflammation limited by AMPK and pseudo-starvation. Nature. 2013;493:346-355.

19. Jensen PR, Peitersen T, Karlsson M, et al. Tissue-specific short chain fatty acid metabolism and slow metabolic recovery after ischemia from hyperpolarized NMR in vivo. J Biol Chem. 2009;284:36077-36082.

20. Bieber LL. Carnitine. Annu Rev Biochem. 1988;57:261-283.

21. Todesco L, Bodmer M, Vonwil K, Haussinger D, Krahenbuhl S. Interaction between pivaloylcarnitine and L-carnitine transport into L6 cells overexpressing hOCTN2. Chem Biol Interact. 2009;180:472-477.

22. Holme E, Greter J, Jacobson CE, et al. Carnitine deficiency induced by pivampicillin and pivmecillinam therapy. Lancet. 1989;2:469-473.

23. Brass EP, Hoppel CL. Relationship between acid-soluble carnitine and coenzyme A pools in vivo. Biochem J. 1980;190:495-504.

24. Ullrich KJ, Rumrich G, Kloss S. Reabsorption of monocarboxylic acids in the proximal tubule of the rat-kidney. 1. Transport kinetics of D-lactate, $\mathrm{Na}+$-dependence, pH-dependence and effect of inhibitors. Pflug Arch. 1982;395:212-219.

25. Rebouche CJ. Kinetics, pharmacokinetics, and regulation of L-carnitine and acetyl-L-carnitine metabolism. Ann N Y Acad Sci. 2004;1033:30-41.

26. Eaton S, Fukumoto K, Stefanutti G, Spitz L, Zammit VA, Pierro A. Myocardial carnitine palmitoyltransferase I as a target for oxidative modification in inflammation and sepsis. Biochem Soc Trans. 2003;31:1133-1136. 